\title{
Fach- und Führungskompetenz in einer datengetriebenen und globalisierten Medienwelt
}

\author{
M.A. „International Marketing \& Media Management“ an der Hochschule für \\ Medien, Kommunikation und Wirtschaft (HMKW)
}

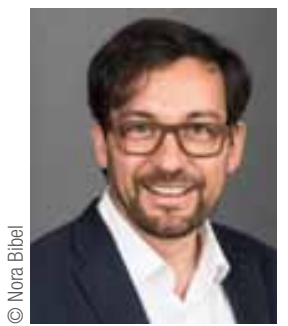

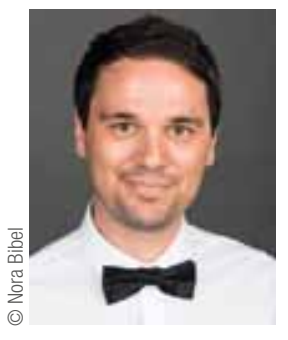

Prof. Dr. Thomas Petzold

HMKW Hochschule für Medien, Kommunikation und Wirtschaft, Berlin

Professor für Medienmanagement

Leiter des Fachbereiches Wirtschaft,

Standort Berlin

t.petzold@hmkw.de

Schlüsselbegriffe: Internationales Marketing | Medienmanagement | Medienökonomie | Masterstudium

Individuelle und globale Managementkenntnisse und -fähigkeiten

Trends wie die Globalisierung und die Automatisierung analytischer, produktiver und dienstleistungsorientierter Aktivitäten führten zu neuen Anforderungen an das Lehren und Lernen in einer wissensbasierten Gesellschaft. Gleichzeitig werden nicht standardisierbare „interaktive Fähigkeiten" (Levy \& Murnane, 2004) wie kreatives Handeln, kritisches Denken, interkulturelle Zusammenarbeit und der Wille zur Innovation insbesondere in der Medienbranche zunehmend als Schlüsselqualifikationen angesehen.

Der Masterstudiengang "International Marketing \& Media Management" (I3M) der HMKW, welcher am Standort Berlin auf Englisch sowie an den Standorten Köln und Frankfurt am Main auf Deutsch angeboten wird, basiert auf einem didaktischen Konzept des forschenden Lernens und einer Auswahl von Inhalten, die den Anforderungen an eine stärkeren Betonung persönlicher und sozialer, insbesondere teamorientierter Kompetenzen Rechnung tragen. Die Studierenden werden in die Lage versetzt, Forschungsprojekte selbstständig zu planen und durchzuführen sowie eng mit verwandten Disziplinen zusammenzuarbeiten.

Aufgrund des Status der HMKW als Fachhochschule bleibt der Gesamtcharakter des Programms jedoch nicht nur forschungs-, sondern ist vor allem auch anwendungsori- entiert: Das Hauptziel des Programms ist es, die Beschäftigungsfähigkeit der Absolventinnen und Absolventen zu fördern, die sich insbesondere durch die Übertragung von fortgeschrittenem theoretischem Wissen und praktischen Fähigkeiten auf typische Anforderungen im beruflichen Umfeld der Medienbranche auszeichnet.

Der Masterstudiengang „International Marketing \& Media Management" vermittelt spezifische Kenntnisse und Fähigkeiten zur Entwicklung von Kompetenzen, die für zukünftige Fach- und Führungskräfte in der global agierenden Marketing- und Medienbranche von wesentlicher Bedeutung sind. Daher konzentriert sich die Lehre und die Forschung auf die Entwicklung dieser persönlichen und beruflichen Kompetenzen:

1. Individuelle Fähigkeiten: Sprachkenntnisse, Präsentationstechniken, psychologische und kulturelle Kenntnisse, interkulturelle Führungstechniken.

2. Theoretische und methodische Fähigkeiten: Analyse wirtschaftlicher, sozialer, technologischer und politischer Entwicklungen aus internationaler Perspektive, basierend auf akademischen Standards und quantitativen Forschungsmethoden.

3. Globale Managementfähigkeiten: Analyse und Weiterentwicklung von Geschäftsstrategien und operativen Managementtechniken, um den individuellen, organisatorischen und wirtschaftlichen Erfolg in globalisierten Märkten zu steigern. 

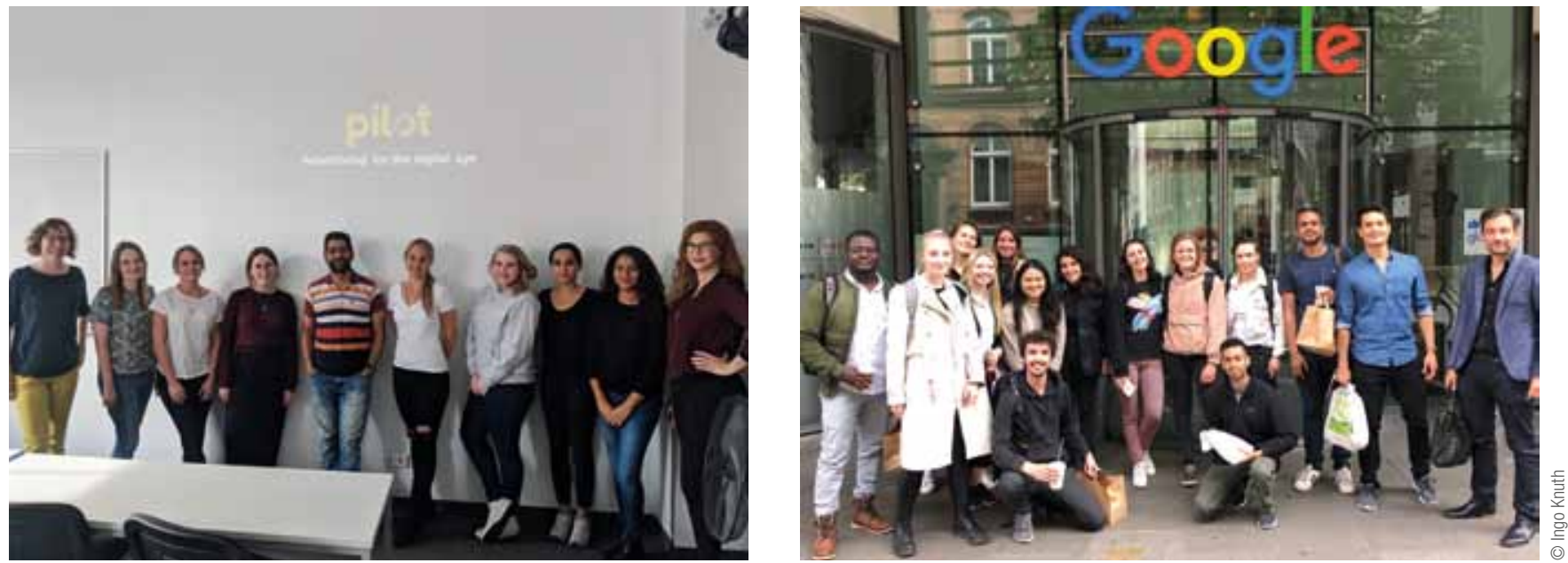

Abbildung 1: Berliner Studierende bei einer Exkursion zu Google Germany und im Rahmen der Abschlussveranstaltung zu Modulen aus dem Bereich Marketingkommunikation mit pilot Media

Basierend auf diesen Elementen werden die Studierenden professionell auf die Bewältigung Managementaufgaben in einer globalisierten und datengetriebenen Medienwelt vorbereitet, zum Beispiel für diese beruflichen Rollen:

- Marketingmanager, Mediamanager, Media Sales Manager

- Business Strategy Manager, Business Development Manager

- Marktforscher, Media Analyst, Business Analyst, Data Scientist

\section{Lernen durch Forschung und Praxisbezug}

Defensives Lernen, um mit Blick auf die Prüfung zu lernen, ist die häufigste, wenn auch kurzsichtige Strategie, um die Herausforderungen eines Studiums zu meistern. Das „Lernen durch Forschung“, d. h. die selbstinitiierte
Aneignung und Bearbeitung von Fragen auf der Grundlage realer Probleme, bietet ein aktiveres und nachhaltigeres Modell. Dabei lernen die Studierenden neben aktuellem Wissen auch Schlüsselqualifikationen in der Vorbereitung auf wissensbasierte betriebliche Wertschöpfung. Gleichzeitig erwerben sie die notwendigen Voraussetzungen für eine spätere akademische Laufbahn.

Einer der Hauptvorteile des Ansatzes "Learning by Research" ist das Potenzial, die Fähigkeit der Studierenden zu fördern, komplexe Probleme aus dem Marketing- und Medienbereich eigenständig und lösungsorientiert anzugehen sowie die Ergebnisse methodisch fundiert, jedoch auch pointiert und anschaulich zu präsentieren. Dabei handelt es sich um Kompetenzen, die insbesondere für Manager und Forscher aus der Medienbranche von besonderer Bedeutung sind. (Knuth/ Petzold, 2019) Zudem können praktische Probleme aus dem wirklichen Leben von den Studierenden mit selbst entwickelten und modifizierten Methoden formuliert, verarbeitet und wis-

\section{Tabelle 1: Modulstruktur I3M}

\begin{tabular}{|c|c|c|c|c|c|c|c|}
\hline \multicolumn{2}{|c|}{ Grundlagenmodule } & \multicolumn{2}{|c|}{ Internationales Marketing } & \multicolumn{2}{|c|}{ Medienmanagement } & \multicolumn{2}{|c|}{ Datenerhebung- und -analyse } \\
\hline$\square$ & Wirtschaftsenglish & $\square$ & Einführung in das Internationale Ma & rketin & g und Medienmanagement & $\square$ & Methoden / Statistik \\
\hline$\square$ & Medienrecht & $\square$ & $\begin{array}{l}\text { Klassische Marketing } \\
\text { Kommunikation }\end{array}$ & $\square$ & $\begin{array}{l}\text { Medienmanagement- } \\
\text { Theorie }\end{array}$ & $\square$ & Marktforschung \\
\hline$\square$ & $\begin{array}{l}\text { Media Asset } \\
\text { Management }\end{array}$ & $\square$ & $\begin{array}{l}\text { Online Marketing } \\
\text { Kommunikation }\end{array}$ & $\square$ & Medienökonomie & $\square$ & $\begin{array}{l}\text { Business Intelligence und } \\
\text { Data Science }\end{array}$ \\
\hline$\square$ & Technologische Trends & $\square$ & Konsumentenverhalten & $\square$ & Emerging Media Markets & & \\
\hline$\square$ & Interkulturelle Führung & $\square$ & Preis- und Vertriebsmanagement & $\square$ & Digitale Transformation & & \\
\hline$\square$ & Medienpsychologie & & & & & & \\
\hline$\square$ & Medienproduktion & & & & & & \\
\hline
\end{tabular}




\section{Service - Forschung + Lehre}

\section{Tabelle 2: Grundlagenmodule}

\begin{tabular}{l|l|l} 
Nr. & Modul & Inhalt \\
\hline Grundlagenmodule & Wirtschaftsenglish & Mündliche und schriftliche Geschäftskommunikation \\
\hline 1 & Medienrecht & Urheberrecht, Datenschutz und andere ethisch-juristische Fragen \\
\hline 2 & Media Asset Management & Konsolidierung von Daten/Dokumenten und Formaten/Versionen \\
\hline 3 & Technologische Trends & Digitalisierung, Konvergenz, neue Technologien \\
\hline 4 & Interkulturelle Führung & Managementfähigkeiten in einer globalisierten Welt \\
\hline 5 & Medienpsychologie & Psychologische Grundlagen des Medienkonsums \\
\hline 6 & Medienproduktion & Planung und Produktion von plattformübergreifenden Medieninhalten \\
\hline 7 & &
\end{tabular}

\section{Tabelle 3: Marketing- und Medienmanagement Module}

\begin{tabular}{l|l|l} 
Nr. & Modul & Inhalt \\
Internationales Marketing und Medienmanagement \\
\hline 1 & $\begin{array}{l}\text { Einführung in das Internationale } \\
\text { Marketing und Medienmanagement }\end{array}$ & $\begin{array}{l}\text { Grundlagen des Medienmanagements, Einführung in das internationale Marketing, Markt- und } \\
\text { Kampagnenanalyse }\end{array}$ \\
\hline Internationales Marketing & $\begin{array}{l}\text { Massenmediale Marketingkommunikation in klassischen Medien (TV, Print, Radio, OOH), Grund- } \\
\text { lagen der Mediaplanung }\end{array}$ \\
\hline 2 & Klassische Marketingkommuni- & kation
\end{tabular}

\section{Tabelle 4: Methodenorientierte Module}

\begin{tabular}{l|l|l} 
Nr. & Modul & Inhalt \\
\hline \multicolumn{2}{l}{ Datenerhebung- und -analyse } & Deskriptive Statistik, multivariate Statistik, Einführung in die Datenvisualisierung \\
\hline 10 & Methoden / Statistik & $\begin{array}{l}\text { Theoretische Hintergründe und moderne Methoden der Markforschung, Konzeption und Durch- } \\
\text { führung von empirischen Forschungsprojekten }\end{array}$ \\
\hline 11 & Marktforschung & $\begin{array}{l}\text { Daten- und Wissensmanagement, deskriptive Analysen, Datenvisualisierung, Datenmodellie- } \\
\text { rung, Machine Leaning }\end{array}$ \\
\hline 12 & $\begin{array}{l}\text { Business Intelligence und Data } \\
\text { Science }\end{array}$ &
\end{tabular}


senschaftlich aufgearbeitet werden. Dieser Aspekt wird durch die Integration von Gastrednern aus namhaften Medien-, Marketing- und Technologieunternehmen, durch die Organisation von Exkursionen und die Durchführung von geschäftsrelevanten Fallstudien und Forschungsprojekten gemeinsam mit international tätigen sowie lokalen Partnern, wie zum Beispiel pilot Media, Initiative Media, Google Germany GmbH, Facebook, Xing SE, eSport-Bund Deutschland e.V., Goodgame Studios, GmbH, ProSiebenSat.1 Media AG, OTTO Group, Berliner Sparkasse oder Hertha BSC GmbH, konkret gefördert.

\section{Struktur und Inhalt des Studiengangs}

Das Studienprogramm ist präsenzbasiert und auf vier Studiensemester mit insgesamt 120 Credit Points ausgelegt. Die Module sind in zwei Gruppen unterteilt - grundlegende und fachspezifische Module. In den Grundlagenmodulen, von denen drei Veranstaltungen gewählt werden können, sollen vor allem übergreifende fachliche Kompetenzen sowie persönliche Kompetenzen gestärkt werden. Die Vermittlung von theoretischen und methodischen Fachkompetenzen erfolgt im Rahmen spezifischer Grundlagen- und Anwendungsmodule zum Marketing- und Medienmanagement sowie innerhalb des Blockes „Datenerhebung- und -analyse“, welcher der Vermittlung und Anwendung empirischer Forschungsmethoden dient.

Die Grundlagenmodule vermitteln Kenntnisse und Fähigkeiten, die für eine weitere berufliche Laufbahn qualifizieren. Sie dienen als grundlegende Bausteine, die für alle akademischen oder beruflichen Spezialisierungen nützlich sind.

Absolventinnen und Absolventen von medienzentrierten Masterstudiengängen müssen auf ein Arbeitsumfeld vorbereitet sein, in dem die Fähigkeit zur Teilnahme an internationaler und kulturspezifischer Kommunikation sowie Kenntnisse über rechtliche Fallstricke vorausgesetzt werden. Dies gilt insbesondere dann, wenn sehr spezifische Fach- oder aber Führungspositionen angestrebt werden. Daher bieten drei Module eine erweiterte Unterstützung zur Stärkung dieser oft entscheidenden Fähigkeiten: Wirtschaftsenglisch, Interkulturelle Führung und Medienrecht.

Gleiches gilt für technisches Know-how im theoretischen und praktischen Sinne: Operative, aber auch betriebswirtschaftliche Arbeit in medienorientierten Umgebungen erfordert die Fähigkeit, die Wirkungsweisen von verschiedenen Medientechnologien zu verstehen sowie mit innen umzugehen. Zur Stärkung dieser Kompetenzen stehen vier Module zur Auswahl, die sich auf diese wichtigen Fähigkeiten konzentrieren: Media Asset Management, Technologische Trends, Medienpsychologie und Medienproduktion.

Die fachspezifischen Module des Masterstudiengangs 'International Marketing \& Media Management' sind in drei Gruppen unterteilt:
- Internationales Marketing: ein Einführungsmodul plus vier Anwendungs- und Vertiefungsmodule

- Medienmanagement: das gleiche Einführungsmodul plus vier Anwendungs- und Vertiefungsmodule

- Datenerhebung- und -analyse: drei methodische, stark empirisch orientierte Module

Diese Module bilden den Kern des gesamten Studiengangs. Sie konzentrieren sich auf wissenschaftlich und beruflich relevante Fächer der Marketing- und Medienmanagementtechniken mit einer internationalen Perspektive, wobei der Schwerpunkt auf methodische Kompetenzen hinsichtlich Datenerhebung, -analyse und -visualisierung liegt, da diese Kenntnisse und Fähigkeiten auf dem Arbeitsmarkt für Marketing- und Medienmanager sehr gefragt sind. Sie dienen als Rahmen für die Forschungsprojekte in verschiedenen Modulen sowie als Vorbereitung für die Masterarbeit.

\section{Zulassungsvoraussetzungen}

Um einerseits möglichst unmittelbar ein hohes fachliches Niveau in der Lehre und der Forschung zu erreichen und andererseits Studierenden mit vielfältigen inhaltlichen und kulturellen Hintergründen den Zugang zum Masterstudiengang I3M zu ermöglichen, werden formale und fachliche Zulassungsvoraussetzungen definiert. So wird ein sehr guter Universitäts- oder Fachhochschulabschluss in allgemeiner Betriebswirtschaftslehre oder in einem anderen wirtschafts- oder sozialwissenschaftlichem Fach vorausgesetzt. Grundsätzlich müssen mindestens 20 Kreditpunkte in management- oder methodenorientierten Fächern erworben worden sein. Die Auswahl der Kandidatinnen erfolgt mittels persönlicher Vorstellungsgespräche und Wissenstests. Am Standort Berlin, wo der Masterstudiengang in englischer Sprache angeboten wird, werden zudem entsprechende Englischkenntnisse erwartet.

\section{Literatur}

HMKW (2018): Infoflyer M.A. Internationales Marketing und Medienmanagement, https://www.hmkw.de/fileadmin/media/downloads/pdfs/infoflyerstudiengaenge_master/neu_18_04/HMKW_MA-Programmes_I3M.pdf 26.09.2019

Knuth, I./Petzold, T. (2019): Solution-Oriented Media Management Research: A Meta Study to Nurture the Future Paradigm of the Field European, Konferenzbeitrag, Jahrestagung der European Media Management Association (EMMA), Limassol / Zypern, 07.06.2019

Levy, F. \& Murnane, R.J. (2004): The new division of labor. How Computers are creating the next job market. Princeton University Press (also see http:// atc21s.org/)

\section{Weitere Informationen}

Homepage: https://www.hmkw.de/hochschule/fachbereiche/wirtschaft/studiengaenge/ma-internationales-marketing-und-medienmanagement/ Instagram: @memhmkw Facebook: @memhmkw Twitter: @mementumblog 